\title{
A subset of the Hermitian surface
}

\author{
Giorgio Donati Nicola Durante
}

full screen

\section{Abstract}

In this paper we define a ruled algebraic surface of $\mathrm{PG}\left(3, q^{2}\right)$, called a hyperbolic $\mathcal{Q}_{F}$-set and we prove that it is contained in the Hermitian surface of $\mathrm{PG}\left(3, q^{2}\right)$. Also, we characterise a hyperbolic $\mathcal{Q}_{F}$-set as the intersection of two Hermitian surfaces.

Keywords: Hermitian surface, collineation.

MSC 2000: 51E20, 05B25.

\section{Introduction}

Let $\mathcal{P}_{A}$ and $\mathcal{P}_{B}$ be the pencils of lines with vertices two distinct points $A$ and $B$ in $\mathrm{PG}\left(2, q^{2}\right)$. Let $\alpha_{F}$ be the involutory automorphism of $G F\left(q^{2}\right)$ given by $x \in G F\left(q^{2}\right) \mapsto x^{q} \in G F\left(q^{2}\right)$ and let $\Phi$ be an $\alpha_{F}$-collineation between $\mathcal{P}_{A}$ and $\mathcal{P}_{B}$. If $\Phi$ does not map the line $A \vee B$ onto the line $B \vee A$, then the set points of intersections of corresponding lines under $\Phi$ is called a $\mathcal{C}_{F}$-set (see [3], [4]). If $\Phi$ maps the line $A \vee B$ onto the line $B \vee A$, then the set of points of intersections of corresponding lines under $\Phi$ is called a degenerate $\mathcal{C}_{F}$-set (see [5]).

Every $\mathcal{C}_{F}$-set has $q^{2}+1$ points, it is of type $(0,1,2, q+1)$ with respect to lines of PG $\left(2, q^{2}\right)$ and every $(q+1)$-secant line intersects such a set in a Baer subline. The $(q+1)$-secant lines number $q-1$ and all contain a common point $C$ not on the $\mathcal{C}_{F}$-set. Those lines, together with the lines $C \vee A$ and $C \vee B$, form a Baer subpencil. The point $C$ is called the centre of the $\mathcal{C}_{F}$-set. Also, every $\mathcal{C}_{F}$-set is projectively equivalent to the algebraic curve with equation

$$
x_{1} x_{2}^{q}-x_{3}^{q+1}=0 .
$$

Under the André-Bruck-Bose representation of $\mathrm{PG}\left(2, q^{2}\right)$ in $\mathrm{PG}(4, q)$ these subsets correspond to three-dimensional elliptic quadrics contained in suitable hyperplanes of $\mathrm{PG}(4, q)$. 
Every degenerate $\mathcal{C}_{F}$-set has $2 q^{2}+1$ points, it is of type $\left(1,2, q+1, q^{2}+1\right)$ with respect to lines of $\operatorname{PG}\left(2, q^{2}\right)$ and every $(q+1)$-secant line intersects such a set in a Baer subline. Also, every degenerate $\mathcal{C}_{F}$-set is the union of the line $A \vee B$ and a Baer subplane meeting the line $A \vee B$ in a Baer subline. Every degenerate $\mathcal{C}_{F}$-set is projectively equivalent to the algebraic curve with equation

$$
x_{3}\left(x_{1} x_{2}^{q-1}-x_{2}^{q}\right)=0 .
$$

The points $A$ and $B$ are called the vertices of a $\mathcal{C}_{F}$-set (degenerate or not).

Observe that the construction of a $\mathcal{C}_{F}$-set (degenerate or not) is a variation of Steiner's projective construction of conics.

In a similar way, we obtain an algebraic surface of $\mathrm{PG}\left(3, q^{2}\right)$ by using a variation of Steiner's projective generation of hyperbolic quadrics.

\section{Definition and properties}

Let $a$ and $b$ be two skew lines of the projective space PG $\left(3, q^{2}\right)$ and let $\mathcal{P}_{a}$ and $\mathcal{P}_{b}$ be the pencils of planes with axes $a$ and $b$. Let $\Phi$ be an $\alpha_{F}$-collineation between $\mathcal{P}_{a}$ and $\mathcal{P}_{b}$; the set of points of intersection of corresponding planes under $\Phi$ is called a hyperbolic $\mathcal{Q}_{F}$-set. In [3] it is proved that every hyperbolic $\mathcal{Q}_{F}$-set of $\mathrm{PG}\left(3, q^{2}\right)$ is projectively equivalent to the algebraic surface with equation

$$
x_{1} x_{4}^{q}-x_{2} x_{3}^{q}=0 .
$$

The lines $a$ and $b$ are called the axes of the hyperbolic $\mathcal{Q}_{F}$-set.

Every hyperbolic $\mathcal{Q}_{F}$-set has $\left(q^{2}+1\right)^{2}$ points and it is the union of $q^{2}+1$ skew lines, each a transversal of $a$ and $b$. These lines, together with $a$ and $b$, are all the lines contained in a hyperbolic $\mathcal{Q}_{F}$-set.

In the following two propositions we investigate the intersection of a hyperbolic $\mathcal{Q}_{F}$-set with lines and planes of $\operatorname{PG}\left(3, q^{2}\right)$.

Proposition 2.1. Every line of $\mathrm{PG}\left(3, q^{2}\right)$ intersects a hyperbolic $\mathcal{Q}_{F}$-set in $0,1,2, q+$ 1 or $q^{2}+1$ points. The $(q+1)$-secant lines intersects a hyperbolic $\mathcal{Q}_{F}$-set in a Baer subline.

Proof. Let $\mathcal{Q}$ be a hyperbolic $\mathcal{Q}_{F}$-set defined by an $\alpha_{F}$-collineation $\Phi$ between the pencils of planes with axes two skew lines $a$ and $b$ of $\operatorname{PG}\left(3, q^{2}\right)$. For a line $\ell$ of $\mathrm{PG}\left(3, q^{2}\right)$, four cases are distinguished.

(1) Either $\ell=a$ or $\ell=b$.

In this case $\ell$ is a $\left(q^{2}+1\right)$-secant line. 





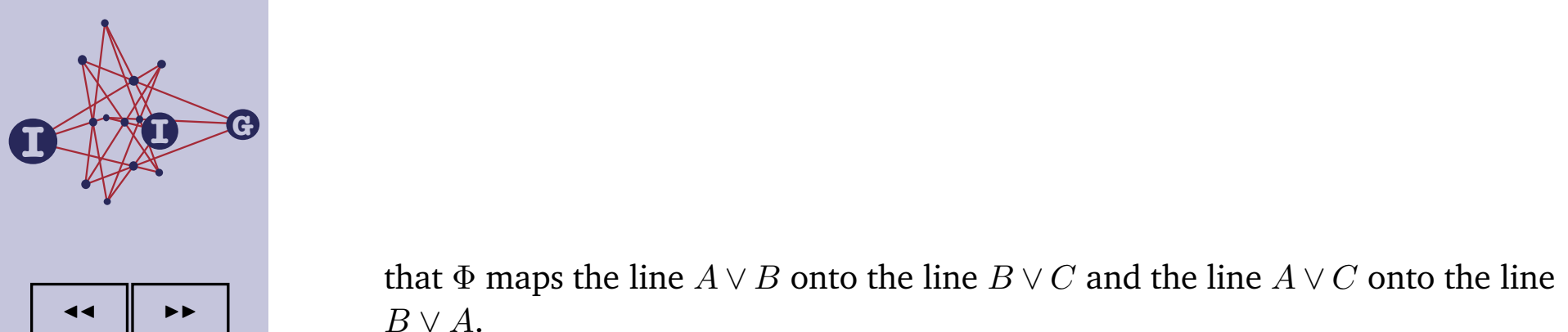
$B \vee A$.

Proposition 2.6. The centres $C_{i}$ of the $q^{2}-1 \mathcal{C}_{F}$-sets $\mathcal{C}_{i}$ are on a common line.

Proof. Let $A=\ell \cap a$ and $B=\ell \cap b$. Let $a_{i}=A \vee C_{i}$ and let $b_{i}=B \vee C_{i}$. We will prove that the line $\left(a \vee a_{i}\right) \cap\left(b \vee b_{i}\right)$ is independent of $i$ and hence contains all points $C_{i}$.

The collineation $\Phi$ maps the plane $a \vee a_{k}$ to the plane $b \vee \ell$ and the plane $a \vee \ell$ to the plane $b \vee b_{k}$ for every $k$, since $\Phi$ induces on $\pi_{k}$ a collineation between pencils of lines with vertices $\pi_{k} \cap a$ and $\pi_{k} \cap b$ which maps $a_{k}=A \vee C_{k}$ onto $\ell=A \vee B$ and $\ell$ onto $b_{k}=B \vee C_{k}$. It follows that $a \vee a_{i}=a \vee a_{j}$ and $b \vee b_{i}=b \vee b_{j}$. The assertion follows.

\section{Hyperbolic $\mathcal{Q}_{F}$-sets and Hermitian surfaces}

A Hermitian surface of $\operatorname{PG}\left(3, q^{2}\right)$ is the set $\mathcal{H}$ of all absolute points of a nondegenerate unitary polarity. It has $\left(q^{2}+1\right)\left(q^{3}+1\right)$ points, and every line of $\mathrm{PG}\left(3, q^{2}\right)$ intersects $\mathcal{H}$ in $1, q+1$ or $q^{2}+1$ points. The $(q+1)$-secant lines each intersect $\mathcal{H}$ in a Baer subline. Every plane of $\mathrm{PG}\left(3, q^{2}\right)$ intersects $\mathcal{H}$ either in a Hermitian curve or in a Baer subpencil.

In [4] it is shown that every Hermitian curve of $\operatorname{PG}\left(2, q^{2}\right)$ contains $\mathcal{C}_{F}$-sets. In the following proposition we prove that every Hermitian surface of $\mathrm{PG}\left(3, q^{2}\right)$ contains hyperbolic $\mathcal{Q}_{F}$-sets.

Proposition 3.1. Let $\mathcal{H}$ be a Hermitian surface of $\mathrm{PG}\left(3, q^{2}\right)$ and let $a$ and $b$ be two skew lines contained in $\mathcal{H}$. Then there exists a hyperbolic $\mathcal{Q}_{F}$-set with axes a and $b$ contained in $\mathcal{H}$.

Proof. Let $u$ be the polarity associated with $\mathcal{H}$. Let $\alpha$ be a plane of the pencil with axis $a$. Since $a$ is contained in $\mathcal{H}$, it follows that $u(\alpha)$ is on $a$. Hence the following map may be defined:

$$
\Phi: \alpha \in \mathcal{P}_{a} \longmapsto b \vee u(\alpha) \in \mathcal{P}_{b}
$$

Since $\Phi$ is an $\alpha_{F}$-collineation, the set of points of intersection of corresponding planes under $\Phi$ is a hyperbolic $\mathcal{Q}_{F}$-set, say $\mathcal{Q}$, of $\operatorname{PG}\left(3, q^{2}\right)$. Also, for every plane $\alpha \in \mathcal{P}_{a}$, the line $\Phi(\alpha) \cap \alpha=(b \vee u(\alpha)) \cap \alpha$, contained in $\mathcal{Q}$, joins the two points points $u(\alpha)$ and $\alpha \cap b$, which are conjugate with respect to the polarity $u$, and hence is contained in $\mathcal{H}$. Therefore $\mathcal{Q}$ is contained in $\mathcal{H}$. 



